\title{
Semi-physical nonlinear circuit model with device/physical parameters for HEMTs
}

\author{
HIROSHI OTSUKA ${ }^{1}$, TOSHIYUKI OISHI ${ }^{1}$, KOJI YAMANAKA ${ }^{1}$, MATTIAS THORSELL ${ }^{2}$, \\ KRISTOFFER ANDERSSON ${ }^{2}$, AKIRA INOUE ${ }^{1}$, YOSHIHITO HIRANO ${ }^{1}$ AND ILTCHO ANGELOV ${ }^{2}$
}

\begin{abstract}
A nonlinear circuit model (NCM) with physical parameters is proposed for direct simulation of the RF characteristics of GaN high-electron-mobility transistors (GaN HEMTs) on the basis of device structure. The physical equations are used for the construction of the model in order to connect strongly the model parameters with the device/physical parameters. Hyperbolic tangent functions are used as the model equations to ensure good model convergence and rapid simulation (short simulation time). The usefulness of these equations is confirmed by technology computer aided design (TCAD) simulation. The number of model parameters for the nonlinear components $\left(I_{d s}, C_{g s}, C_{g d}\right)$ is reduced to 17 by using common physical parameters for modeling the drain current and capacitance. The accuracy of this model is verified by applying to GaN HEMTs. The modeled I-V and capacitance characteristics agree well with the measurement data over a wide voltage range. Furthermore, this model can be used for the accurate evaluation of S-parameters and large-signal RF characteristics.
\end{abstract}

Keywords: Transistor models, GaN HEMT, Simulation, Power amplifiers, Physical modeling, Microwave

Received 26 August 2010; Revised 4 January 2011

\section{INTRODUCTION}

High-power amplifiers (HPAs) that include GaN high-electron-mobility transistors (GaN HEMTs) have potential applications in radar systems, satellite communication systems, cellular base stations, etc. Efforts have been made to improve the performance of $\mathrm{GaN}$ field effect transistor (FETs) on the basis of the vast experience accumulated over the years $[1-17]$. While several high-power, high-efficiency HPAs have been reported in the past [1-5], there is considerable scope for improvement of the HPA performance; this is because of the immaturity of the existing GaN technologies. Therefore, concurrent development of device and circuit design methods is desired. Empirical nonlinear circuit models (ENCMs) are widely used in circuit design. ENCMs are fast and converge well and give an accurate fit for $\mathrm{DC}$ and $\mathrm{RF}$ characteristics [6-9]. However, such circuit models are not suitable for use in device design because there is no explicit relationship between the model parameters and the device/ physical parameters. Moreover, a relatively large number of parameters must be extracted. On the other hand, physical models involving device/physical parameter calculations are too slow to be used in circuit simulations; further, these models do not converge well during RF simulation because of the complicated and exhaustive calculation for some boundary regions [10-13]. Therefore, simple ENCMs constructed using device/physical parameters are preferred. These models may help improve the device performance by contributing to an

\footnotetext{
${ }^{1}$ Information Technology R\&D Center, Mitsubishi Electric Corporation 5-1-1 Ofuna, Kamakura, Kanagawa 247-8501, Japan. Phone: + 81467412692. ${ }^{2}$ Department of Microtechnology and Nanoscience (MC2), Microwave Electrics Laboratory, GHz Centre, Chalmers University of Technology, Gothenburg SE-41296, Gothenburg, Sweden.

Corresponding author:

H. Otsuka

Email: Otsuka.Hiroshi@bc.MitsubishiElectric.co.jp
}

increase in the FET efficiency in practical circuit applications and facilitating device performance optimization in specific applications.

It is a widely accepted fact that hyperbolic tangent ( $\tanh$ ) functions can be used for a satisfactory description of the $I-V$ and capacitance characteristics of HEMT devices $[15,16]$. Such tanh functions have been used to describe the $I-V$ characteristics of GaN HEMTs as well $[8,9]$. The large-signal (LS) HEMT model [7] is a highly accurate model whose convergence is sufficiently high for it to be used in circuit design simulation. However, the model parameters, as in the case of every empirical device model, are used for reproducing the shape of the $I-V$ curves, gm, capacitance characteristics, etc., rather than for device design.

In this paper, we propose a new model based on ENCM equations designed using device/physical parameters. We modified the model to obtain a direct connection with the device/ physical parameters. The model parameters are similar to the parameters used in device design. Furthermore, the number of model parameters for the nonlinear components $\left(I_{d s}, C_{g s}, C_{g d}\right)$ can be reduced to 17 because the same physical parameters are used to describe the $I-V$ and capacitance characteristics. This model is extended to a GaN HEMT, and the model parameters are extracted; the modeled $I-V$ and capacitance characteristics are in excellent agreement with the measurement results. Moreover, with this model, accurate determination of the S-parameters and LS RF characteristics is possible.

\section{MODEL}

\section{A) Drain-current model}

To construct a drain-current model, we started from the following physical equation:

$$
I_{d s}=q n v_{e}
$$


where $I_{d s}, q, n$, and $v_{e}$ are the drain current, electron charge, electron concentration of the two-dimensional electron gas (2DEG) and electron velocity, respectively. Figure 1 shows a schematic cross section of an HEMT. The electrons with $n$ and $v_{e}$ travel from the source to the drain in the channel.

Next, we need to find a suitable function to model $n$ and $v_{e}$. It is known that $n$ is controlled mainly by the gate voltage $\left(V_{g s}\right)$, and the tanh function was successfully used to model $n=f\left(\tanh \left(V_{g s}\right)\right)[15,16]$. In order to make it possible to adjust the model for various cases, the tanh function for $n$ was expressed as

$$
\begin{gathered}
n=A\left(\frac{1+\tanh B}{2}\right) \\
B=C_{1}\left(V_{g s}-D\right)+C_{2}\left(V_{g s}-D\right)^{2}+C_{3}\left(V_{g s}-D\right)^{3}
\end{gathered}
$$

where $A, B, C_{1}, C_{2}, C_{3}$, and $D$ are constant. $C_{2}$ and $C_{3}$ are polynomial coefficients related to the carrier density.

For $v_{e}$, we adopted the following equation [18], which depends only on the drain voltage $\left(V_{d s}\right)$, that is, $v_{e}=$ $g\left(\tanh \left(V_{d s}\right)\right)$, because the electron accelerates owing to the laterally electronic field between the gate and drain, as shown in Fig. 1:

$$
v_{e}=E \tanh \frac{V_{d s}}{F}
$$

where $E$ and $F$ are constants. We confirmed the effectiveness of equations (2)-(4) by technology computer aided design (TCAD) simulation, where we employed the $2 \mathrm{D}$ device simulator (Silvaco ATLAS [19]). Figures 2 and 3 show the curves of the tanh functions fitted to the TCAD simulation results. $A, C_{1}, C_{2}, C_{3}$, and $D$ in equations (1) and (2) were $1.35 \mathrm{e} 12 \mathrm{~cm}^{-2}, 0.4 \mathrm{~V}^{-1}, 0.0 \mathrm{~V}^{-2}, 0.02 \mathrm{~V}^{-3}$, and $0.8 \mathrm{~V}$, respectively. The values of $E$ and $F$ in equation (4) are $2.52 \mathrm{e} 7 \mathrm{~cm} / \mathrm{s}$ and $3.5 \mathrm{~V}$, respectively. For both $n$ and $v_{e}$, the model using the tanh function agreed very well with the TCAD simulation results. Therefore, equations (2)-(4), which are designed on the basis of tanh functions, can be used to express the characteristics of $n$ and $v_{e}$.

Finally, we represented the drain-current model by substituting equations (2)-(4) in equation (1) and assigning the

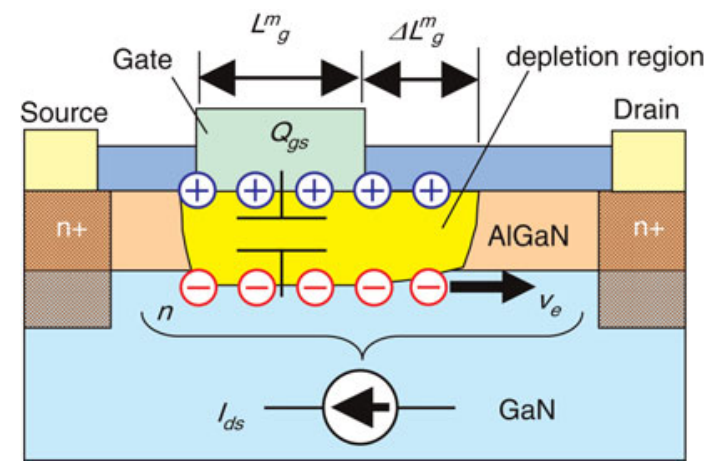

Fig. 1. Schematic cross section of HEMT model.

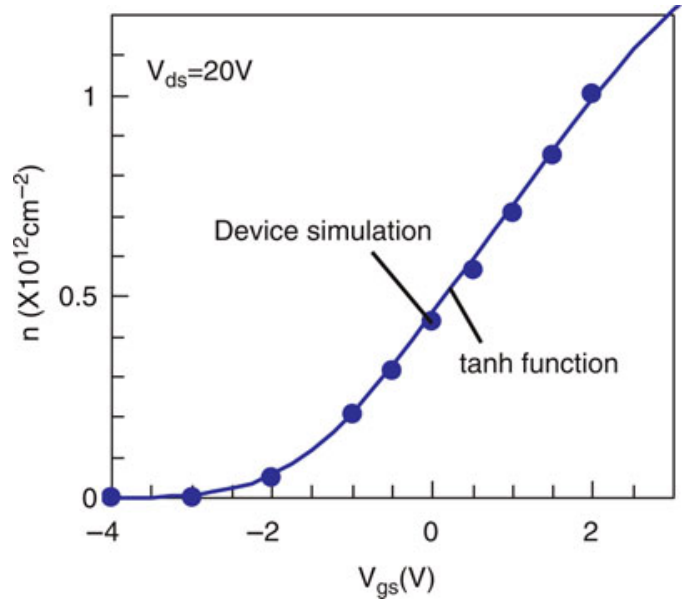

Fig. 2. Curve of tanh function fitted to TCAD simulation data for $n$.

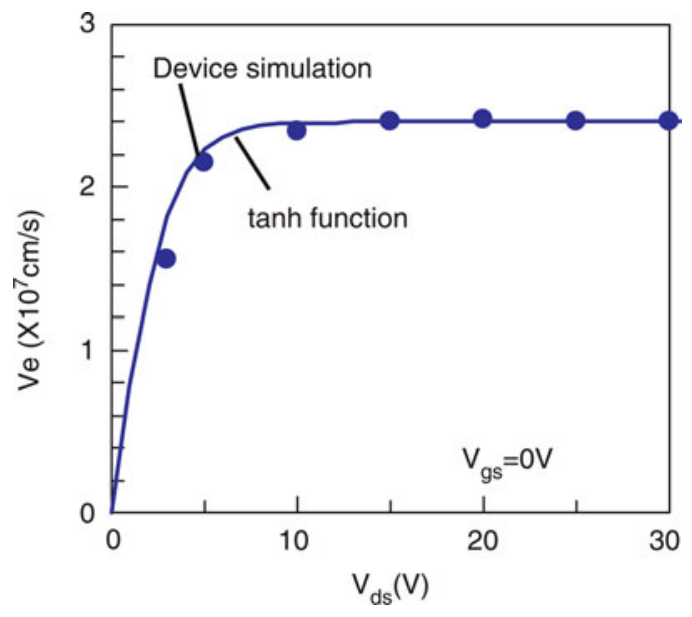

Fig. 3. Curve of tanh function fitted to TCAD simulation data for $v_{e}$.

parameters $A-F$ to the device parameters:

$$
\begin{gathered}
I_{d s}=I_{\max }\left(\frac{1+\tanh \psi}{2}\right) \tanh \frac{2 V_{d s}}{V_{k n e e}} \\
\psi=P_{1}\left(V_{g s}-V_{p}-\frac{1}{P_{1}}\right)+P_{2}\left(V_{g s}-V_{p}-\frac{1}{P_{1}}\right)^{2} \\
+P_{3}\left(V_{g s}-V_{p}-\frac{1}{P_{1}}\right)^{3} \\
P_{1}=\frac{2 g_{m p k}}{I_{\max }}
\end{gathered}
$$

where $I_{\text {max }}, V_{\text {knee }}, V_{p}$, and $g_{m p k}$ are the maximum drain current, knee voltage, pinch-off voltage, and maximum transconductance, respectively. $P_{2}$ and $P_{3}$ are polynomial coefficients related to the carrier density and will allow us to adjust the current shape according to the channel profile. $P_{1}$ consists of two device parameters $\left(I_{\max }, g_{m p k}\right)$. Four of the input parameters $\left(I_{\text {max }}, V_{\text {knee }}, V_{p}\right.$, and $\left.g_{m p k}\right)$ except $P_{2}$ and $P_{3}$ can be used as the device parameters, which are normally used in the device design and are familiar to the device engineers. 


\section{B) Capacitance model}

The gate-source capacitance $\left(C_{g s}\right)$ model is constructed from the following physical-based equation:

$$
C_{g s}=\frac{\partial Q_{g s}}{\partial V_{g s}}=q \frac{\partial n}{\partial V_{g s}}\left(L_{g}^{m}+\Delta L_{g}^{m}\right)
$$

where $Q_{g s}$ and $L_{g}^{m}$ are the gate charge and metallurgical-gate length, respectively. $\Delta L_{g}^{m}$ is defined as the extension length of the depletion region, as shown in Fig. 1. Usually, $Q_{g s}$ corresponds to the charge of the parallel plate with an effective gate length $L_{g}^{m}$. However, an extension of the depletion region from the gate edge to the drain should be considered, because a large $V_{g d}$ was applied to the GaN HEMTs. We consider an extension of the depletion region, expanding $L_{g}^{m}$ to $\left(L_{g}^{m}+\right.$ $\left.\Delta L_{g}^{m}\right)$. We used the tanh function, which limits the maximum value to $\Delta L_{g}^{m}$, represented by

$$
\Delta L_{g}^{m}=G \tanh \left(H V_{g d}\right)
$$

where $G$ and $H$ are constants. $\Delta L_{g}^{m}$ depends only on $V_{g d}$, because the depletion region extends mainly laterally. The shape of the $\Delta L_{g}^{m}-V_{g d}$ curve obtained by the TCAD simulation can be fitted well by equation (9), as shown in Fig. 4. $G$ and $H$ are $1.05 \mu \mathrm{m}$ and $110 \mathrm{~V}$, respectively. For $n$ in equation (8), the same expression as that for $\mathrm{n}$ in the drain-current model in equation (5) can be used. This reduces the number of model parameters.

Finally, the model $C_{g s}$ is represented by

$$
\begin{aligned}
C_{g s}= & C_{g s p i}+C_{g s o} I_{\max } L_{g}\left(\frac{d \psi / d V_{g s}}{2 \cosh ^{2} \psi}\right) \\
& \times\left[1+R_{L g} \tanh \left(\alpha_{L g} V_{g d}\right)\right]
\end{aligned}
$$

where $C_{g s o}, R_{L g}, \alpha_{L g}$, and $C_{g s p i}$ are the gate-source capacitance, the ratio of $L_{g}^{m}+\Delta L_{g}^{m}$ to $L_{g}^{m}$, gate extension coefficient, and the parasitic capacitance between gate and source, respectively.

In the case of the gate-drain capacitance $\left(C_{g d}\right)$, the charge model $\left(Q_{g s}\right)$ is not sufficient to produce a useful $C_{g d}$ expression from the physics. Because the $C_{g d}$ is smaller than one-tenth of

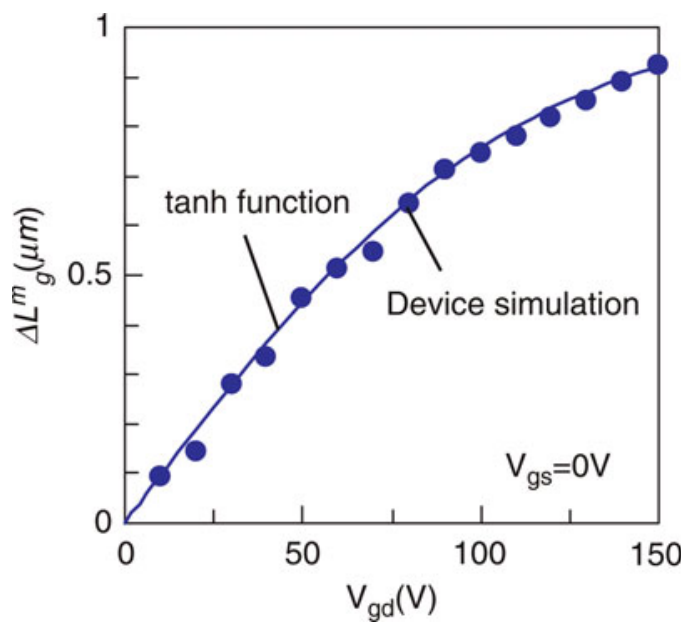

Fig. 4. Curve of the tanh function fitted to TCAD simulation data for $\Delta L_{g}^{m}$.
$C_{g s}$, and more accuracy model of the $Q_{g s}$ is necessary. So we used the conventional expression of the inversely proportional function dependent only on $V_{g d}[13]$ instead, which yields

$$
C_{g d}=\frac{C_{g d o}}{\left(1-\left(V_{g d} / V_{b i}\right)\right)^{M S C G D}}+C_{g d p i}
$$

where $V_{b i}, C_{g d p i}$, and MSCGD are the built-in voltage, parasitic capacitance, and capacitance coefficient for $C_{g d}$, respectively. $C_{g d o}$ is the gate-drain capacitance at $V_{g d}=0$.

\section{C) Relationship between model parameters and physical parameters}

Figures 5(a) and 5(b) show the device structure for $\mathrm{GaN}$ HEMTs and the band diagram under the gate electrode. In Fig. 5(a), $L_{s g}$ and $L_{g d}$ are the gate-source distance and the gate-drain distance, respectively. $\sigma, v_{s}$, and $\mu$ are the polarization charge, saturation electron velocity, and electron mobility, respectively. $R_{c}, R_{L s g}, R_{L g}$, and $R_{L g d}$ are the contact resistance, source-gate resistance, gate resistance, and gatedrain resistance, respectively. In Fig. 5 (b), $\Phi_{b}, d$, and $\Delta E_{C}$ are the gate work function, AlGaN layer thickness, and conduction band difference between the AlGaN layer and the GaN channel. These physical parameters are useful for device design, particularly in terms of performance optimization.

This model can be connected easily to the physical parameters due to the explicitly defined expressions of the device parameters. Device parameters can be estimated from physical parameters by using relational equations. Equations (19) and (20) used in this model are simple and make it easy to understand the relation between device parameters and physical parameters. The equations can be also easily implemented in standard, default CAD LS models.

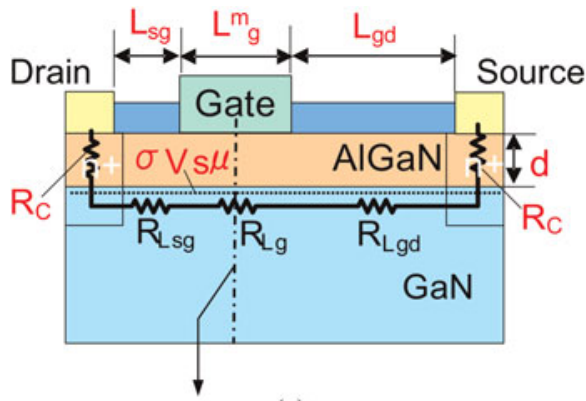

(a)

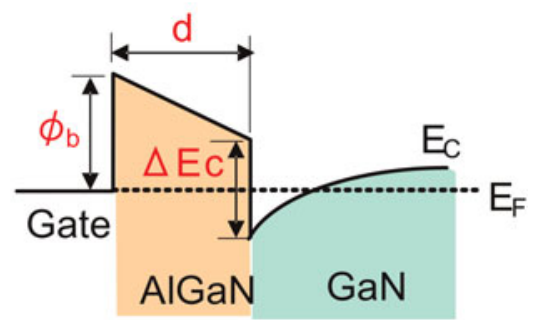

(b)

Fig. 5. (a) Device structure for GaN HEMTs and (b) band diagram under gate electrode. 
The maximum drain current $I_{\text {max }}$ is expressed with respect to $\sigma$ and $v_{s}$, as follows [20]:

$$
I_{\max }=q \sigma v_{s}
$$

The knee voltage $V_{\text {knee }}$ is expressed with respect to $I_{\text {max }}$ and the on-state resistance $R_{o n}$, which indicates the slope of the drain current in the linear region:

$$
V_{\text {knee }}=R_{\text {on }} I_{\text {max }}
$$

where

$$
\begin{aligned}
R_{o n} & =R_{L s g}+R_{L g}+R_{L g d}+2 R_{c} \\
& =\frac{\left(L_{s g}+L_{g d}+L_{g}^{m}\right)}{q \sigma \mu}+2 R_{c}
\end{aligned}
$$

In equation (14), the product $q \sigma \mu$ represents the electrical conductivity of $2 \mathrm{DEG}$.

Accounting for the source resistance $R_{s}$, the maximum transconductance $g_{m p k}$ is expressed as [20]

$$
g_{m p k}=\frac{g_{m i}}{1+g_{m i} R_{s}}
$$

where $g_{m i}$ is the intrinsic transconductance without parasitic elements. $g_{m i}$ is [20] given by

$$
g_{m i}=\frac{v_{s} \varepsilon_{G} \varepsilon_{0}}{d}
$$

where $\varepsilon_{G}$ and $\varepsilon_{\mathrm{o}}$ are the GaN permittivity and vacuum permittivity, respectively. Similar to equation (14), $R_{s}$ in equation (15) is expressed as

$$
R_{s}=\frac{\left(L_{s g}+(1 / 2) L_{g}^{m}\right)}{q \sigma \mu}
$$

The pinch-off voltage, $V_{p}$, is defined based on [21], as shown in Fig. 5(b):

$$
V_{p}=\phi_{b}-\Delta E_{c}-\frac{q \sigma}{\varepsilon_{A G} \varepsilon_{0}} d
$$

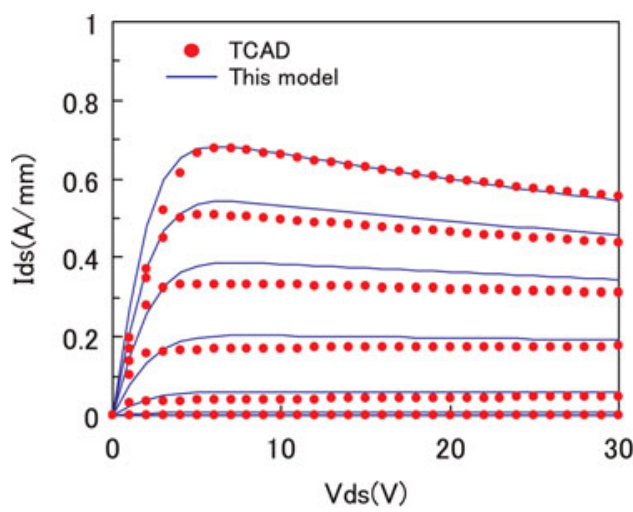

(a)
In this way, it is easy to relate the model parameters to the physical parameters in this model. The device parameters can be obtained by substituting the values of the physical parameters in equations (12)-(18). In addition, the drain current can be calculated by using the device parameters and equations (5)-(7). In other words, this model can calculate directly the $I-V$ characteristics from the device structure.

Figure 6 shows the $I-V$ characteristics of this model and the TCAD simulation. In this model, the self-heating effect was accounted for by substituting the following equations in equation (5):

$$
\begin{gathered}
I_{\max }=I_{\text {maxo }}\left(1-T_{c I p k} \Delta T_{\text {chan }}\right) \\
\Delta T_{\text {chan }}=\left(T_{a m b}-25\right)+R_{\text {therm }}\left(I_{d s} V_{d s}\right)
\end{gathered}
$$

$I_{\text {maxo }}$ is the maximum drain current in the absence of the selfheating effect. $T_{c I p k}$ is the temperature coefficient of the drain current. $\Delta T_{\text {chan }}, T_{a m b}$, and $R_{\text {therm }}$ are the channel temperature, ambient temperature, and thermal resistance, respectively. In the TCAD simulation, Poisson's equation, the current continuity equation, and the heat-transfer equation were solved simultaneously for self-heating effect. The physical parameters used in this simulation are summarized in Table 1 . The simulation conditions are $V_{g s}=-5$ to $2 \mathrm{~V}\left(1 \mathrm{~V}\right.$ steps) and $V_{d s}=$ $0-30 \mathrm{~V}(1 \mathrm{~V}$ steps). The models agreed well with the TCAD simulation. This result shows that this model accurately predicts the $I-V$ characteristics from physical parameters.

\section{EXPERIMENTAL VERIFICATION}

Figure 7 shows an equivalent circuit for GaN HEMT that implements the drain-current model and the capacitance model. The equivalent circuit consists of linear elements and nonlinear elements. The linear elements and the nonlinear elements represent a parasitic part and an intrinsic part in a transistor, respectively. The nonlinear elements are divided into three components which are the drain current $\left(I_{d s}\right)$, nonlinear capacitances $\left(C_{g s}, C_{g d}, C_{d s}\right)$, and a diode $\left(D_{g s}, D_{g d}\right)$. The linear circuits are made up of parasitic inductances $\left(L_{g}, L_{d}, L_{s}\right)$, parasitic capacitances $\left(C_{p g}, C_{p d}\right)$, and parasitic resistances $\left(R_{g}\right.$, $R_{d}, R_{s}$ ). This model accounts for the self-heating effect and the charge-trapping effect. A simple RC network is employed as a

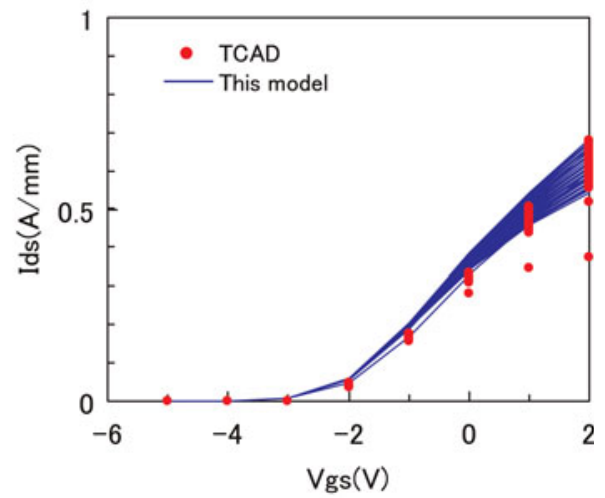

(b)

Fig. 6. Simulated and modeled $I-V$ characteristics of GaN HEMT: (a) $I_{d s}-V_{d s}$ and (b) $I_{d s}-V_{g s}$ curves. 
Table 1. Physical parameters.

\begin{tabular}{lllll}
\hline No. & Name & Description & Value & Unit \\
\hline 1 & $\mathrm{Al}_{x}$ & Al content of AlGaN layer & 0.2 & \\
2 & $d$ & AlGaN layer thickness & 27 & $\mathrm{~nm}$ \\
3 & $V_{b i}$ & Built-in voltage & 1.3 & $\mathrm{~V}$ \\
4 & $\sigma$ & Polarization charge & $6.6 \mathrm{e} 12$ & $\mathrm{~cm}^{-2}$ \\
5 & $V_{\mathrm{s}}$ & Saturation electron velocity & $1 \mathrm{e} 7$ & $\mathrm{~cm} / \mathrm{s}$ \\
6 & $L_{S G}$ & Gate-source distance & 1 & $\mu \mathrm{m}$ \\
7 & $L_{G D}$ & Gate-drain distance & 2 & $\mu \mathrm{m}$ \\
8 & $L^{m} g$ & Gate length & 1 & $\mu \mathrm{m}$ \\
9 & $R_{c}$ & Contact resistance & 2.3 & $\Omega \mathrm{mm}$ \\
10 & $\mu$ & Electron mobility & 1400 & $\mathrm{~cm} / \mathrm{V} / \mathrm{s}$ \\
11 & $\kappa_{\mathrm{GaN}}$ & Thermal conductivity of $\mathrm{GaN}$ at $300 \mathrm{~K}$ & 1.25 & $\mathrm{~W} / \mathrm{cm} / \mathrm{K}$ \\
12 & $\kappa_{\mathrm{SiC}}$ & Thermal conductivity of $\mathrm{SiC}$ at $300 \mathrm{~K}$ & 4.9 & $\mathrm{~W} / \mathrm{cm} / \mathrm{K}$ \\
\hline
\end{tabular}

thermal circuit. We use a series RC network to account for the charge-trapping effect [8]. The expressions for $I_{d s}, C_{g s}$, and $C_{g d}$ in equations (5)-(7), (10), and (11) are applied to the draincurrent model, gate-source capacitance, and gate-drain capacitance.

The model was verified by using a GaN HEMT including an ion implantation doping of Si under a source/drain electrode for low ohmic contacts [14]. The epitaxial layers consisted of $\mathrm{AlGaN} / \mathrm{GaN}$ layers grown on semi-insulating SiC substrates.

The parameter-extraction sequence of our model is simple and straightforward. For $I_{d s}$ parameters $\left(I_{\text {max }}, V_{\text {knee }}, V_{p}, g_{m p k}\right)$, the initial values were extracted from the measurement of $I-V$

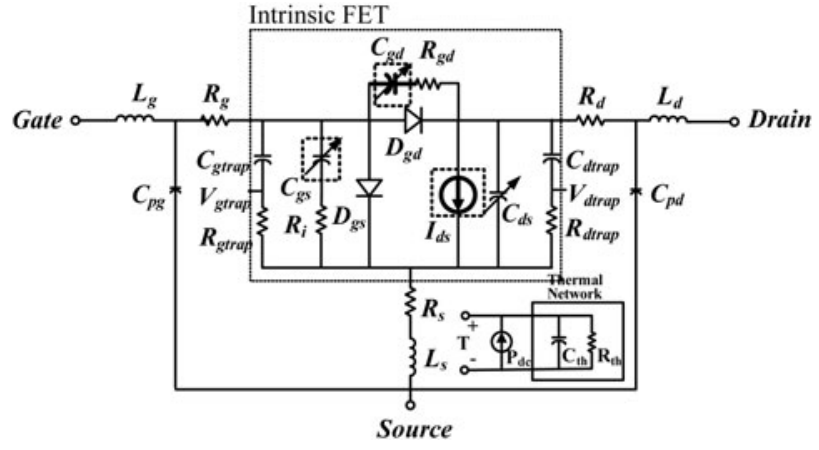

Fig. 7. Equivalent circuit of HEMT.

characteristics. The values can also be calculated from equations (12)-(18). The other parameters were set to zero. Next, the parameters were optimized by a CAD tool.

Parameters $C_{g s}$ and $C_{g d}$ are extracted from multibias $\mathrm{S}$-parameter data. Firstly, intrinsic S-parameters were obtained by de-embedding parasitic elements. Parasitic elements were extracted by means of the cold FET method [17]. Next, converting the S-parameters to Y-parameters, $C_{g s}$ and $C_{g d}$ were obtained [22]. Finally, the parameters were optimized in order to minimize the error between the measurement and model data. In the optimization, the parameters related to the carrier density were the same as the values used in parameter $I_{d s}$. We can also use the values estimated

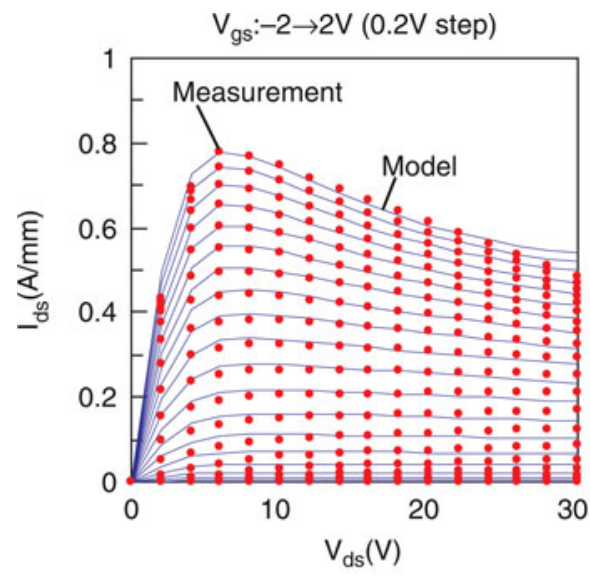

(a)

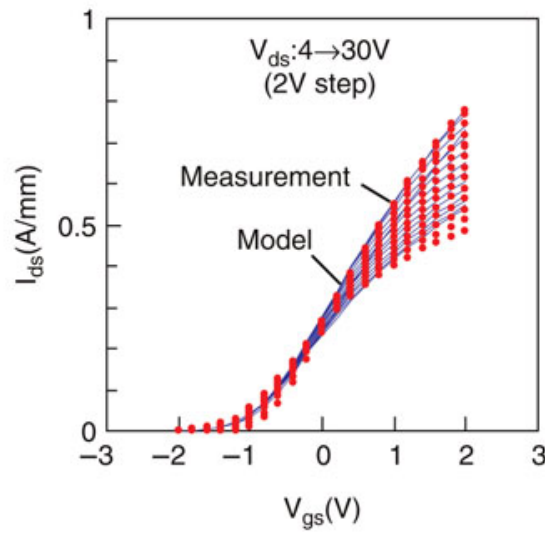

(b)

Fig. 8. Measured and modeled $I-V$ characteristics of GaN HEMT: (a) $I_{d s}-V_{d s}$ and (b) $I_{d s}-V_{g s}$ curves.

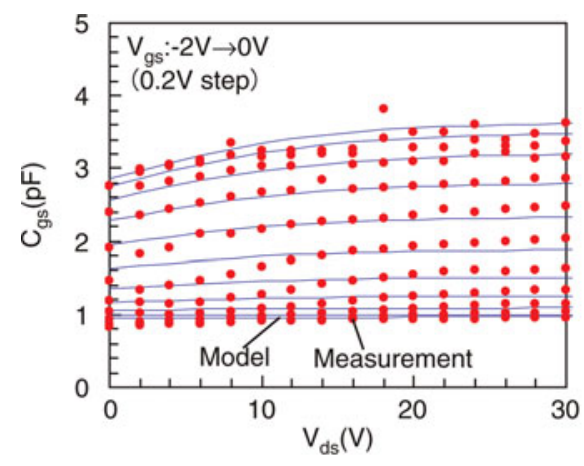

(a)

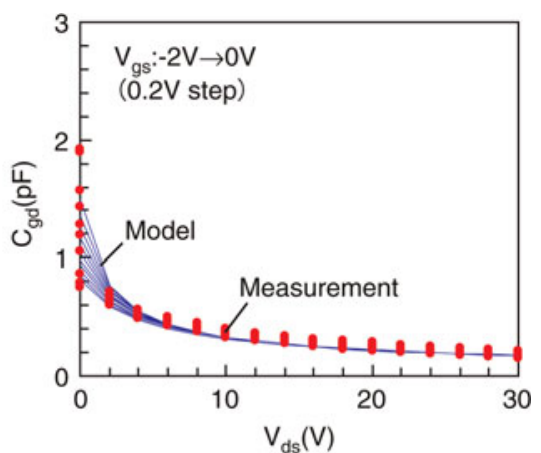

(b)

Fig. 9. Measured and modeled capacitance characteristics of GaN HEMT: (a) $C_{g s}-V_{d s}$ and (b) $C_{g d}-V_{d s}$ curves. 


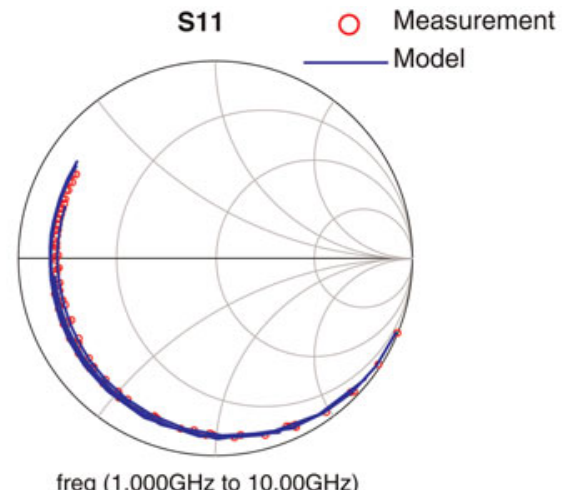

(a)

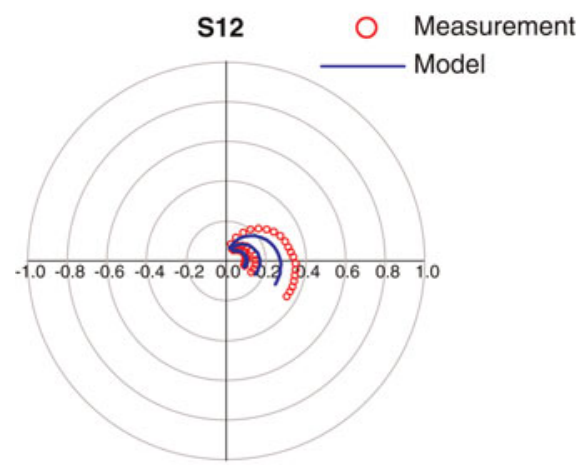

freq $(1.000 \mathrm{GHz}$ to $10.00 \mathrm{GHz})$

(c)

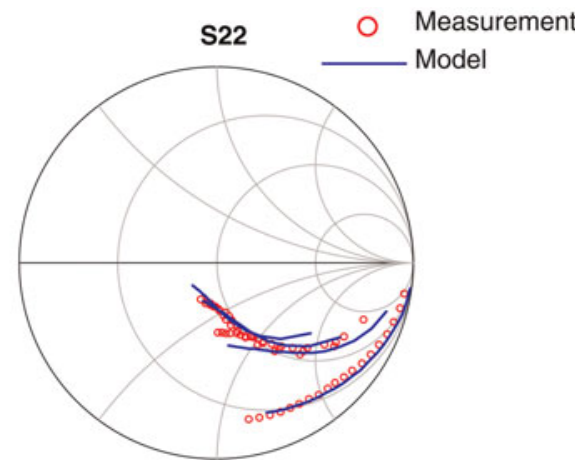

freq $(1.000 \mathrm{GHz}$ to $10.00 \mathrm{GHz})$

(b)

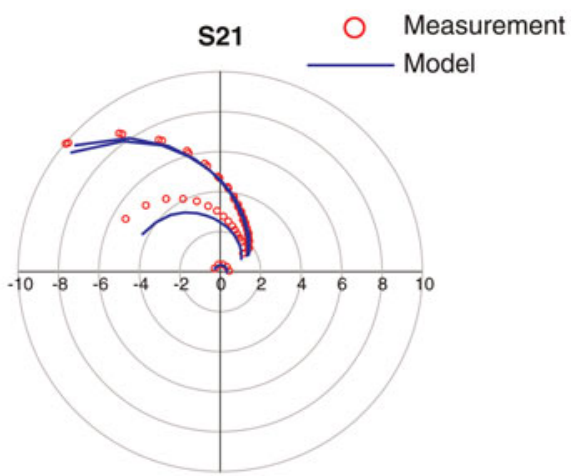

freq $(1.000 \mathrm{GHz}$ to $10.00 \mathrm{GHz})$

(d)

Fig. 10. Measured and modeled multibias $S$-parameter: (a) $S_{11}$, (b) $S_{22}$, (c) $S_{12}$, and (d) $S_{21}$ of the $0.5-m m$ GaN HEMT for $1-10$ GHz at $V_{g s}=-2-1$ V (1 V steps) and $V_{d s}=20 \mathrm{~V}$.

Pout

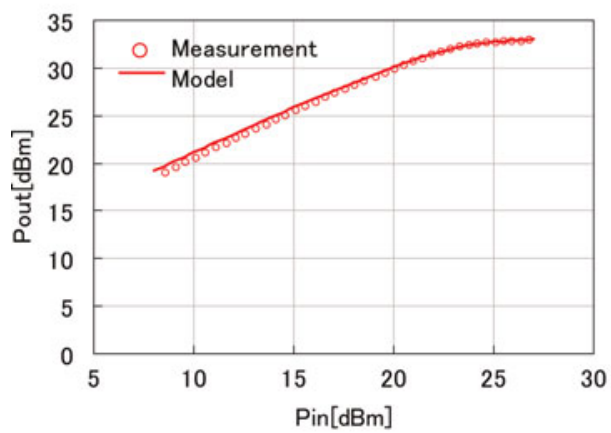

(a)

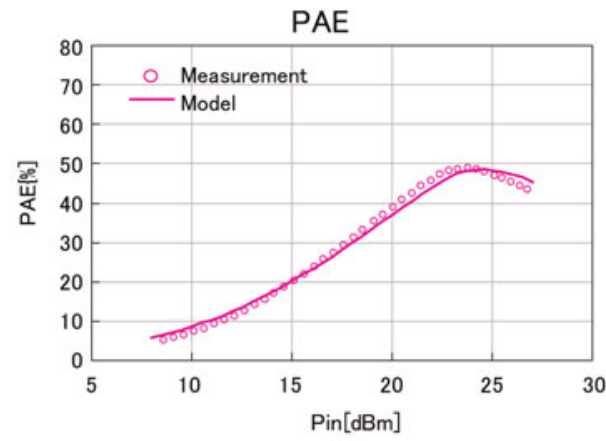

(c)

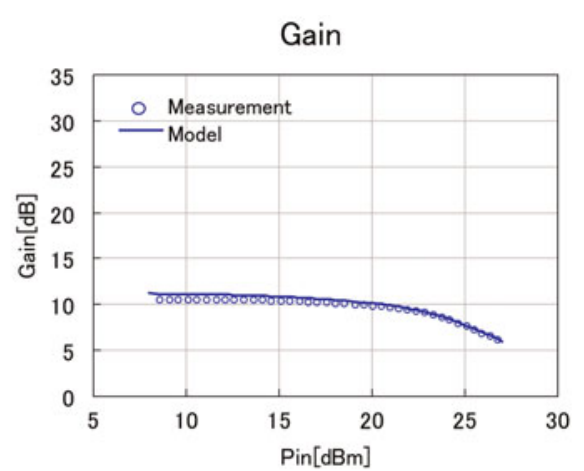

(b)

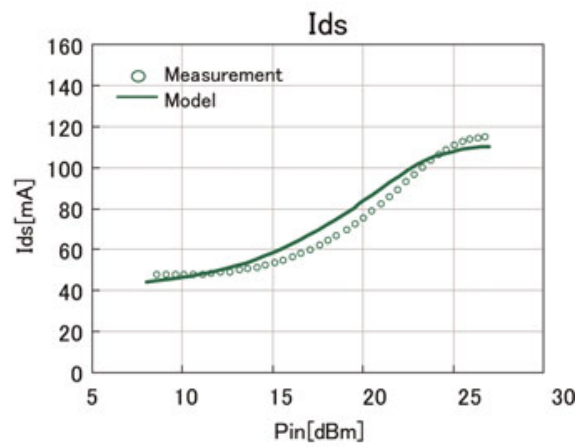

(d)

Fig. 11. Measured (symbols) and modeled (solid lines) (a) output power, (b) gain, (c) power-added efficiency, and (d) drain current of the o.5-mm GaN HEMT for $9.6 \mathrm{GHz}$ at $V_{d s q}=30 \mathrm{~V}$ and $I_{d s q}=50 \mathrm{~mA}$. 
Table 2. Model parameters for nonlinear components.

\begin{tabular}{|c|c|c|c|c|}
\hline No. & Name & Description & Value & Unit \\
\hline 1 & $I_{\max }$ & Maximum drain current & 1.1 & A \\
\hline 2 & $V_{\text {knee }}$ & Knee voltage & 6.8 & $\mathrm{~V}$ \\
\hline 3 & $G_{m p k}$ & Maximum transconductance & 0.34 & S \\
\hline 4 & $V_{p}$ & Pinch-off voltage & -0.91 & $\mathrm{~V}$ \\
\hline 5 & $P_{2}$ & $\begin{array}{l}\text { Polynominal coefficient for carrier } \\
\text { density }\end{array}$ & -0.035 & $1 / \mathrm{V}^{2}$ \\
\hline 6 & $P_{3}$ & $\begin{array}{l}\text { Polynominal coefficient for carrier } \\
\text { density }\end{array}$ & 0.093 & $1 / \mathrm{V}^{3}$ \\
\hline 7 & $R_{\text {therm }}$ & Thermal resistance & 10 & ${ }^{\circ} \mathrm{C} / \mathrm{W}$ \\
\hline 8 & $T_{c l p k}$ & $\begin{array}{l}\text { Temperature coefficient of drain } \\
\text { current }\end{array}$ & -0.003 & \\
\hline 9 & $C_{g s o}$ & Gate source capacitance coefficient & 5.38 & $\mathrm{pF}$ \\
\hline 10 & $C_{g s p i}$ & Parasitic gate source capacitance & 0.94 & $\mathrm{pF}$ \\
\hline 11 & $L^{m} g$ & Metallurgical gate length & 1 & $\mu \mathrm{m}$ \\
\hline 12 & $R_{L g}$ & Ratio of effective gate length to $L^{m} g$ & 0.41 & \\
\hline 13 & $\alpha_{L g}$ & Coefficient for gate extension & 0.07 & \\
\hline 14 & $\mathrm{Cg} d o$ & Gate drain capacitance coefficient & 1.54 & $\mathrm{pF}$ \\
\hline 15 & $C_{g d p i}$ & Parasitic gate drain capacitance & $1 e-9$ & $\mathrm{pF}$ \\
\hline 16 & $V_{b i}$ & Built in voltage & 1.3 & $\mathrm{~V}$ \\
\hline 17 & MSCGD & Capacitance coefficient for $C_{g d}$ & 0.68 & \\
\hline
\end{tabular}

from the physical parameters as the initial values for the optimization.

The models agreed very well with the measurement data. Figure 8 shows (a) $I_{d s}-V_{d s}$ and (b) $I_{d s}-V_{g s}$ curves. The measured bias conditions were over $V_{g s}$ from -2 to $+2 \mathrm{~V}$ (0.2 V steps) and $V_{d s}$ from o to $30 \mathrm{~V}$ (2 V step). The agreement was obtained over a wide range of $V_{d s}$ from o to $30 \mathrm{~V}$.

Figure 9 shows (a) $C_{g s}-V_{d s}$ and (b) $C_{g d}-V_{d s}$ curves. The measured bias conditions were over $V_{g s}$ from -2 to o $\mathrm{V}$ ( 0.2 $\mathrm{V}$ step) and $V_{d s}$ from o to $30 \mathrm{~V}$ (2 V steps). The model reproduced the tendency of the measurements. The model parameters $C_{g s}$ were equivalent to the model parameters $I_{d s}$ related to carrier density $n$.

Figure 10 shows the measured and modeled multibias S-parameter. The solid line and the dotted line represent the model simulation and the measurement, respectively. Bias conditions were over $V_{g s}$ from -2 to $1 \mathrm{~V}\left(1 \mathrm{~V}\right.$ steps) at $V_{d s}=20 \mathrm{~V}$. The frequency range is from 1 to $10 \mathrm{GHz}$. The agreement was obtained over a wide range of frequencies and bias values. The model accurately simulated small-signal characteristics.

Figure 11 shows the measured and modeled LS RF characteristics of a GaN HEMT with a gate width of $0.5 \mathrm{~mm}$ at 9.6 GHz. The measured bias conditions were at $V_{d s q}=30 \mathrm{~V}$ and $I_{d s q}=100 \mathrm{~mA} / \mathrm{mm}$. The optimum input and output impedances at the fundamental frequency $f o$ and at $2 f o$ were determined based on a load-pull measurement. The model accurately predicted LS RF characteristics. The model parameters for nonlinear components are summarized in Table 2. This model can describe $I_{d s}, C_{g s}$, and $C_{g d}$ based on 17 parameters. $R_{\text {therm }}$ and $T_{\text {cIpk }}$ are the thermal resistance and temperature coefficient of the drain current, respectively. A self-heating effect was included by decreasing $I_{d s}$ in the high-current and high-voltage region by these parameters.

\section{CONCLUSION}

We proposed a semi-physical nonlinear circuit model with device/physical parameters. The model equations were derived from the physical ones. A hyperbolic tangent function was used as the model equation to reproduce the electron concentration and electron velocity calculated by TCAD simulation. The $I-V$ characteristics in the TCAD simulation could be predicted from the physical parameters used in this model.

In the model, the same physical parameters are used to model the $I-V$ and capacitance characteristics, which are related to the electron concentration. Therefore, the model can deal directly with device/physical parameters, and it can be expressed by a very small number of model parameters (17) for nonlinear components $\left(I_{d s}, C_{g s}, C_{g d}\right)$. This model has been easily implemented in LS CAD models, by inserting the physical-based equations into equivalent circuit models.

In the verification, the $\mathrm{LS}$ CAD model parameters were extracted for GaN HEMT. The $I-V$ and capacitance characteristics estimated by the model agreed well with the measurement data. Moreover, this model could be used for the accurate estimation of S-parameters and LS RF characteristics. This model is expected to be a powerful tool for improving the performance of GaN HEMTs because of its usefulness in device and circuit design. In device design, the HEMT characteristics can be estimated on the basis of the physical parameters and device structure.

\section{ACKNDWLEDGEMENTS}

The authors would like to thank Messrs. Noto and Kuwata for his support in measurement of the AlGaN/GaN HEMTs. They would also like to thank Dr. Nakayama for the review of this paper and various useful suggestions.

\section{REFERENCES}

[1] Ohtsuka, H. et al.: Over 57\% efficiency C-band GaN HEMT high power amplifier with internal harmonic manipulation circuits, in 2008 IEEE MTT-S Int. Microwave Symp. Dig. WE1E-03, June 2008.

[2] Mishra, U.K.: GaN-Based RF power devices and amplifiers. Proc. IEEE, 96 (2) (2008), 287-305.

[3] Okamoto, Y.; Wakejima, A.; Ando, Y.; Nakayama, T.; Matsunaga, K.; Miyamoto, H.: 10oW C-band single-chip GaN FET power amplifier. Electron. Lett., 42 (5) (2006), 283-285.

[4] Shigematsu, H. et al.: C-band-340-W and X-band 10o-W GaN power amplifiers with over 50\% PAE, in 2009 IEEE MTT-S Int. Microwave Symp. Dig. TH3A-1, June 2009.

[5] Takagi, K. et al.: Ku-band, AlGaN/GaN-HEMT with over $30 \%$ of PAE, in 2009 IEEE MTT-S Int. Microwave Symp. Dig. WE1C-4, June 2009.

[6] Curtice, W.R.; Ettenberg, M.: A nonlinear GaAs FET model for use in the design of output circuits for power amplifiers. IEEE Trans. Microw. Theory Tech., MTT-33 (12) (1985), 1383-1394.

[7] Angelov, I.; Bengtsson, L.; Garcia, M.: Extensions of the Chalmers nonlinear HEMT and MESFET model. IEEE Trans. Microw. Theory Tech., 44 (10) (1996), 1664-1674.

[8] Angelov, I. et al.: Large-signal modeling and comparison of AlGaN/ GaN HEMTs and SiC MESFETs, in Proc. of Asia-Pacific Microwave Conf. (APMC) 2006, WE4B-1, December 2006.

[9] Deng, J. et al.: Temperature-dependent RF large-signal model of GaN-based MOSFETs. IEEE Trans. Microw. Theory Tech., MTT-56 (12) (2008), 2709-2716. 
[10] Pedro, J.C.: A physics-based MESFET empirical model, in 1994 IEEE MTT-S Int. Microwave Symp. Dig. WE3F-14, May 1994.

[11] Li, M.; Wang, Y.: 2-D analytical model for current-voltage characteristics and transconductance of AlGaN/GaN MODFETs. IEEE Trans. Electron Devices, ED-55 (1) (2008), 261-267.

[12] Koudymov, A. et al.: Analytical HFET I-V model in presence of current collapse. IEEE Trans. Electron Devices, ED-55 (3) (2008), 712-720.

[13] Staudinger, J.; Golio, M.; Woodin, C.; de Baca, M.C.: Considerations for improving the accuracy of large-signal GaAs MESFET models to predict power amplifier circuit performance. IEEE J. Solid-state Circuit, SSC-29 (3) (1994), 366-373.

[14] Suita, M.; Nanjo, T.; Oishi, T.; Abe, Y.; Tokuda, Y.: Ion implantation doping for AlGaN/GaN HEMTs. Phys. Stat. Sol. (c), 3 (6) (2006), $2364-2367$.

[15] Roblin, P.; Kang, S.C.; Morkoc, H.: Analytic solution of the velocitysaturated MOSFET/MODFET wave equation and its application to the prediction of the microwave characteristics of MODFETs. IEEE Trans. Electron Devices, ED-37 (7) (1990), 1608-1622.

[16] Roblin, P.; Rohdin, H.; Hung, C.J.; Chiu, S-W.: Capacitance-voltage analysis and current modeling of pulse-doped MODFETs. IEEE Trans. Electron Devices, ED-36 (11) (1989), 2394-3404.

[17] Golio, M.: Microwave MESFETs and HEMTs. Artech, (1991), 152-170.

[18] Ishihara, N.; Shimizu, T.: A simple equation model for RF MOS FET, Silicon Monolithic Integrated Circuits in RF Systems, 2006, Digest of Papers, 2006 Topical Meeting, 18-20 January San Diego, CA, 2006, 93-96.

[19] Device Simulator Atlas Ver. 5.16.3.R. Atlas User's Manual, Silvaco Int., Santa Clara, CA, 2010.

[2o] Chavarkar, P.; Mishra, U.: Field effect transistors: FETs and HEMTs. Thin Films, 28 (2001), 71-145.

[21] Yin, H.: A physics-based large-signal analytical model for AlGaN/ GaN HFETs, Dissertation, North Carolina State University, 2008.

[22] Dambrine, G.; Cappy, A.; Helidore, F.; Playes, E.: A new method of determining the FET small-signal equivalent circuit. IEEE Trans. Microw. Theory Tech., 36 (7) (1988), 1151-1159.

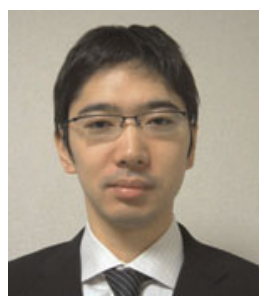

Hiroshi Otsuka received the B.S. and M.S. degrees in physics from Keio University, Tokyo, in 1999 and 2001, respectively. He is with Mitsubishi Electric Corp., working in the field of research and development of microwave amplifiers for radar or telecommunication system from 2001. Mr. Otsuka is a senior member of the Institute of Electronics, Information and Communication Engineers (IEICE) of Japan.

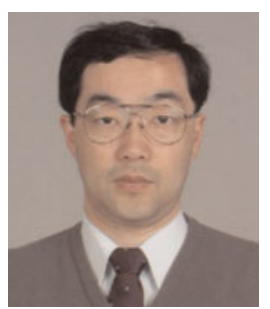

Toshiyuki Oishi was born in Shizuoka, Japan, in 1961. He received the B.E. and M.E. degrees in electrical engineering from Kyoto University, Kyoto, Japan, in 1984 and 1986, respectively. In 2000, he received the Ph.D. degree in ultrafine trench isolation and MOSFET for ULSI from Nagoya University, Nagoya, Japan. In 1986, he joined Central Research Laboratory, Mitsubishi Electric Corporation in Japan. From 1986, he worked on opto-electronic devices using InP related materials, trench isolation, and MOSFET in Si technologies. Since 2001, he has developed on GaN HEMT device and process technologies. From 2008, he joined Information Technology R\&D Center, and started to work on modeling of GaN HEMT. He currently researches the GaN HEMT modeling designed simultaneously both device and circuit. Dr. Oishi is a member of IEEE and the Institute of Electronics, Information and Communication Engineers of Japan.

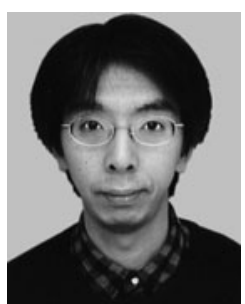

Koji Yamanaka was born in Hyogo, Japan, in 1971. He received the B.S. degree in electric engineering in 1993 and M.S. and Ph.D. degrees in electronic engineering from University of Tokyo, Japan, in 1995 and 1998, respectively. In 1998 he joined Information Technology R\&D Center, Mitsubishi Electric Corporation, where he has been engaged in the development of GaAs low-noise MMIC amplifiers and GaN high-power amplifiers. He received the Best Paper Prize in GAAS2005 for the paper entitled "S and C band Over 100 W GaN HEMT 1-chip High-Power Amplifiers with Cell Division Configuration".

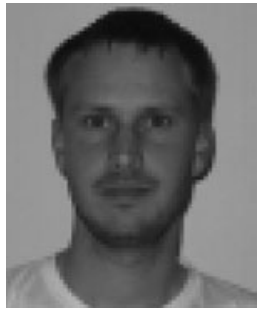

Mattias Thorsell received his M.Sc. degree in electrical engineering from Chalmers University of Technology, Göteborg, Sweden, in 2007. He is currently working towards the Ph.D. degree at Chalmers. His main research topic is characterization and modeling of wide bandgap transistors.

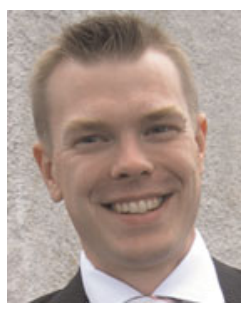

Kristoffer Andersson (S'O3-M'06) received the M.Sc. and Ph.D. degrees in electrical engineering from Chalmers University of Technology, Göteborg, Sweden, in 2001 and 2006, respectively. He currently holds a position as assistant professor at Chalmers University of Technology Göteborg, Sweden. His research interests are in the area of characterization and modeling of wide bandgap transistors.

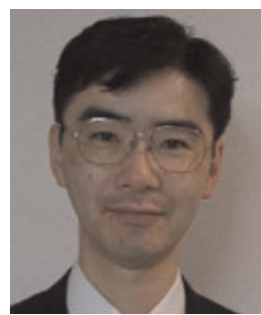

Akira Inoue was born in Osaka, Japan, in 1961 . He received the B.S. and M.S. degrees in physics from the Kyoto University, Japan, in 1984 and 1986, respectively. In 1986 he joined the LSI Laboratory, Mitsubishi Electric Corporation, where he was engaged in the development of GaAs MMIC, microwave power transistors and power amplifiers. He has been developed the first commercial inverse class- $\mathrm{F}$ power amplifier for mobile handsets. In 2005, he received $\mathrm{Ph} . \mathrm{D}$. degree in electrical engineering from Kobe University, Japan on the efficiency enhancement technology in 
microwave high-power semiconductor amplifiers. He was working on the developments of $\mathrm{GaN}$ and HBT power amplifiers at Information Technology R\&D Center. Currently, he is a manager of the mobile phone device development section at High frequency and Optical Device Works. Dr. Inoue is a senior member of the IEEE and the Institute of Electronics, Information and Communication Engineers of Japan (IEICE).

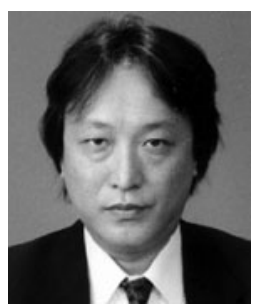

Yoshihito Hirano (M'94-SM'04) received the B.E. and M.E. degrees in material physics, and D.E. degrees in electronic and information engineering from Osaka University, Osaka, Japan, in 1983, 1985, and 2001, respectively. In 1985, he joined Mitsubishi Electric Corporation, Kamakura, Japan. He has been working in research on optical communication equipments, solid-state lasers, nonlinear optics, laser Radars, and microwave photonics. Dr. Hirano is a member of the Japan Society of Applied Physics, the Laser Society of Japan, the Institute of Electrical Engineers of Japan, and the Optical Society of America. He is also a senior member of IEEE Photonics Society.

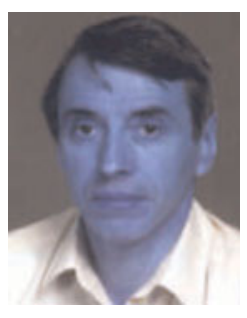

Iltcho Angelov is born in Bulgaria, received M.Sc. in electronics (with Honours) in 1969, Ph.D. in mathematics and physics from Moscow State Univversity in 1973. From 1969-1991 he was with Institute of Electronics, Bulgarian Academy of Sciences (IE BAS) Sofia as Researcher, Research Professor (1982), and Head of Department of Microwave Solid State Devices (1982). Since 1992 he is with Chalmers University, Göteborg, Sweden, as a Research Professor. His research topics are related to FET, HBT modeling, and low-noise and nonlinear circuits design. 